\title{
Lung Ultrasound - A Novel Diagnostic Tool To Phenotype Chronic Lung Allograft Dysfunction?
}

\section{Introduction}

Lung transplantation (LTX) is a well-established treatment in a number of end-stage lung diseases that increases quality of life and survival corresponding to a one-, five-, and ten-year survival rate of $90 \%, 60-70 \%$, and $40-50 \%$, respectively. Most patients die of chronic lung allograft dysfunction (CLAD) which affects approximately $50 \%$ of all lung transplant recipients after five years. Since 2010 growing attention has been paid to the phenotyping of CLAD as bronchiolitis obliterans syndrome (BOS) and restrictive CLAD ( $\mathrm{rCLAD}$ ). BOS is defined as a persistent decline in forced expiratory volume in one second $\left(\mathrm{FEV}_{1}\right)$ of $20 \%$ compared to the average of the two best post-transplant values, whereas rCLAD is defined as a combined decline in $\mathrm{FEV}_{1}$ and total lung capacity (TLC) of more than $10 \%$ compared to the best post-transplant baseline values (Sato M et al. J Heart Lung Transplant. 2011;30:735-42). BOS may respond well to pharmacological treatment, whereas there is not yet any documented rCLAD treatment. Also, in contrast to BOS, rCLAD is associated with pleuroparenchymal fibroelastosis (PPFE) on high-resolution computed tomography (HRCT) and has an inferior survival rate compared to BOS (Verleden SE et al. J Heart Lung Transplant. 2014;33:270-7).

Identification of pleura pathology and fibrosis in interstitial lung diseases, such as idiopathic PPFE, can be identified using lung ultrasound (LUS) in the presence of pleural thickening and the so-called interstitial syndrome (IS), which, in accordance with the international evidence-based guideline for point-of-care LUS, is defined as more than $3 \mathrm{~B}$-lines in more than 2 anterior and/or lateral zones on each hemithorax (Volpicelli G et al. Intensive Care Med. 2012;38:577-91). As a radiation-free and fast procedure which is easily performed with minimal discomfort for the patient, LUS excels in a clinical setting.

Below we describe LUS findings in a lung transplant recipient compatible with PPFE findings on an HRCT, which in combination with lung physiology was in favor of rCLAD.

\section{Case Description}

A male in his mid-fifties with bilateral lung transplant due to alfa 1 antitrypsin deficiency with emphysema experienced unantici-

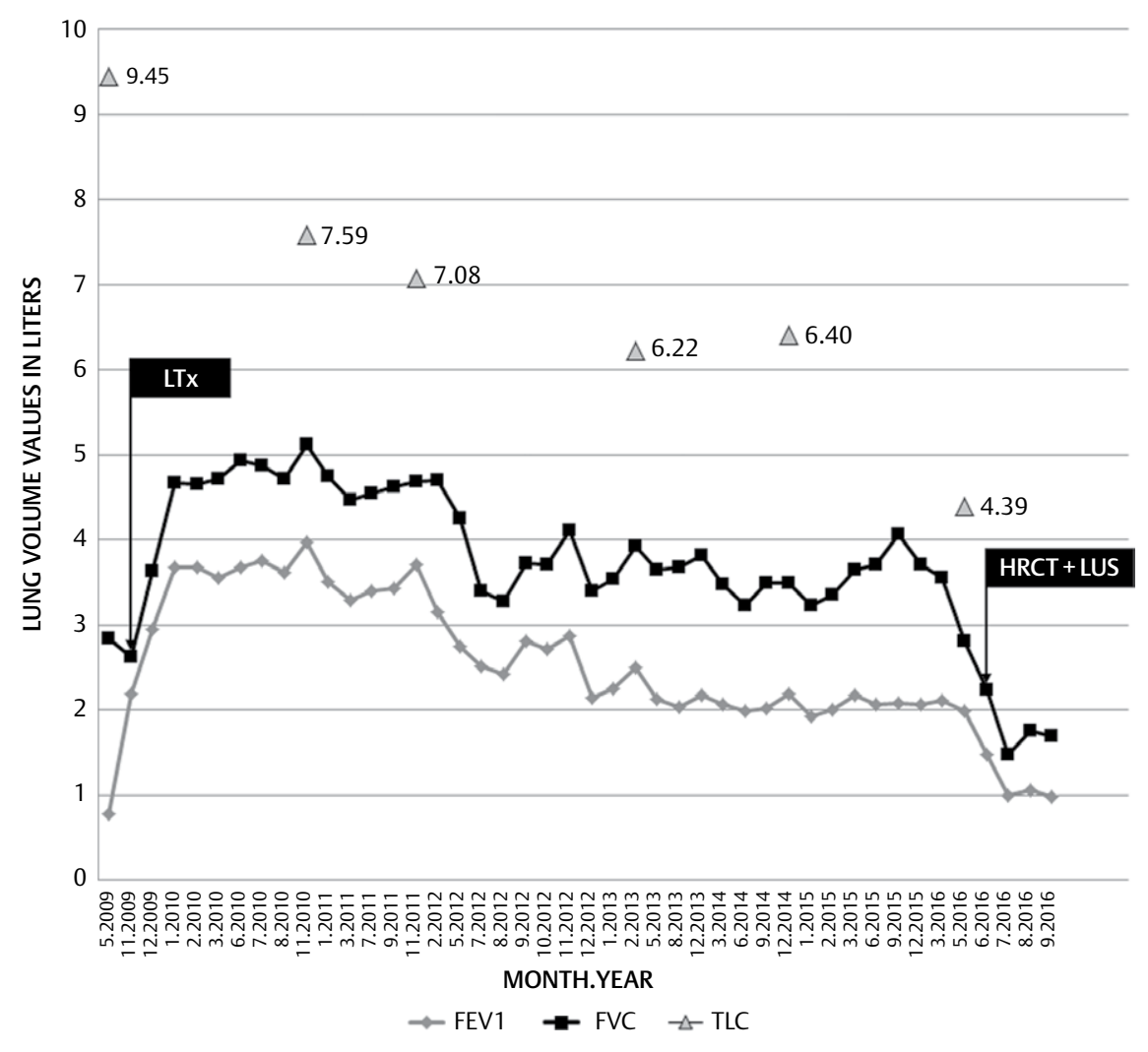

- Fig. 1 Course of pre- and post-transplant lung volume values. Black boxes indicate date of lung transplantation (LTx) and follow-up with high-resolution computed tomography (HRCT) and lung ultrasound (LUS) due to clinical deterioration. FEV1: Forced expiratory volume in one second. FVC: Forced vital capacity. TLC: Total lung capacity. Values for TLC are stated in liters. 


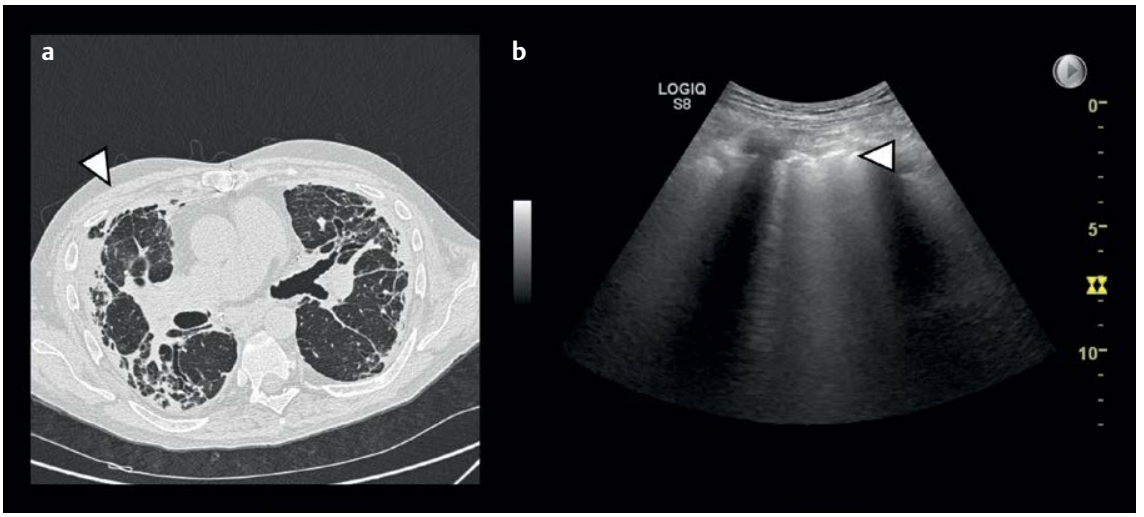

- Fig. 2 a: Axial high-resolution computed tomography (HRCT) image showing upper lobe septal and pleural thickening with fibrotic consolidation compatible with pleuroparenchymal fibroelastosis (PPFE) related restrictive chronic lung allograft dysfunction (rCLAD). White arrow corresponds anatomically to lung ultrasound (LUS) from the anterior part of the right upper lobe, so-called zone R1, in b: LUS clip from the anterior part of the right upper lobe. White arrow indicates a pathological and fragmented pleural line together with confluent parenchymal B-line artifacts.

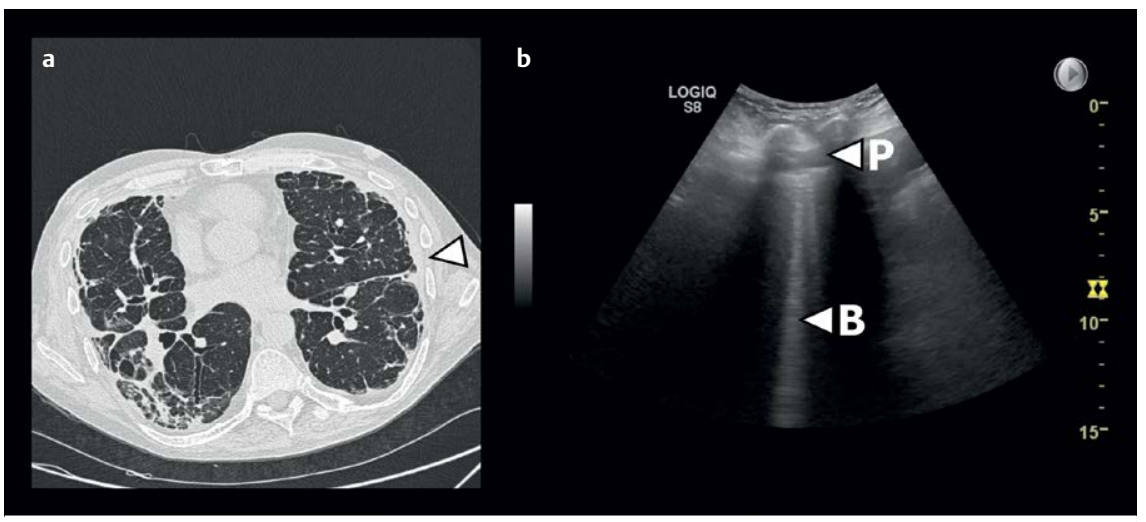

- Fig. 3 a: Axial high-resolution computed tomography (HRCT) image showing pleural thickening as part of pleuroparenchymal fibroelsastosis (PPFE) related restrictive chronic lung allograft dysfunction ( $\mathrm{rCLAD}$ ) corresponding to the left upper lobe. White arrow corresponds anatomically to LUS performed in the lateral part of the left upper lobe, so-called zone $L 3 / 4$, in b: LUS clip from the lateral part of the left upper lobe showing confluent parenchymal B-line artifacts (B) and a thickened hyperechoic pleura (P).

pated and increasing dyspnea at his post-transplant follow-up at 6 years and 9 months. A body plethysmography revealed a significant decline in $\mathrm{FEV}_{1}$ and TLC compared to previous and stable posttransplant values ( $\triangleright$ Fig. 1). An HRCT documented upper lobe pleural and septal thickening with fibrotic consolidation compatible with the radiological appearance of PPFE. On the same day, but following the $\mathrm{HRCT}$, the clinical examination was supplemented with LUS which identified IS and an irregular and thickened pleura line corresponding to the upper lobes as being suggestive of pleuroparenchymal fibrosis. No other etiological explanations (e.g., lung infection, pneumothorax, pleural effusion) of pleuroparenchymal thickening in the upper lobes (Reddy TL et al. Eur Resp J 2012: 40;377-85). Since pleuroparenchymal thickening can be identified using HRCT, the same findings might be extrapolated when assessing patients with LUS. However, a major limitation is that LUS cannot be used to assess the underlying aerated lung tissue. Possible pitfalls could therefore be other conditions with pleural thickening predominantly affecting the upper lobes (e. g., chronic pulmonary aspergillosis (CPA), pulmonary sarcoidosis stage IV, and chronic hypersensitivity pneumonitis (cHP)). Nevertheless, when assessing the thickened pleura in the upper lobes, some signs may possibly be used to differentiate PPFE from other conditions. CPA may cause apical pleural thickening to the same extent as PPFE, but as opposed to PPFE the pleural thickening is relatively homogeneous. When CPA is assessed using LUS, the thickened pleura may thus appear homogeneous with fragmentation being less pronounced. In pulmonary sarcoidosis stage IV and $\mathrm{cHP}$, the pleura may appear thickened and fragmented in the upper lobes. However, based on the study by Reddy et al., we expect the extent of pleural thickening to be more severe in PPFE than in sarcoidosis stage IV and cHP. In studies assessing the use of LUS in interstitial lung diseases, the predominant findings are $\mathrm{B}$-lines and a thickened and fragmented pleura line (Reissig A et al. Respiration 2014;87:179-89). The fragmented pleura line most often appears hyperechoic and not hypoechoic as was the case in the presented case. As such, LUS findings in the form of relatively marked pleural thickening and concomitant fragmentation in the upper lobes may be specific LUS findings compatible with PPFE.

HRCT is a mandatory radiological modality in the examination of CLAD in combination with lung physiology and sometimes invasive diagnostic procedures. However, based on the above-mentioned reflections, LUS may be considered as an alternative add-on diagnostic modality to HRCT in order to support the distinction and phenotyping of CLAD subtypes as BOS and $r C L A D$. Due to the inferior prognosis of $\mathrm{rCLAD}$, a potential association between HRCT verified PPFE and LUS findings suggestive of PPFE in rCLAD patients is also of prognostic interest, and furthermore, sup- 


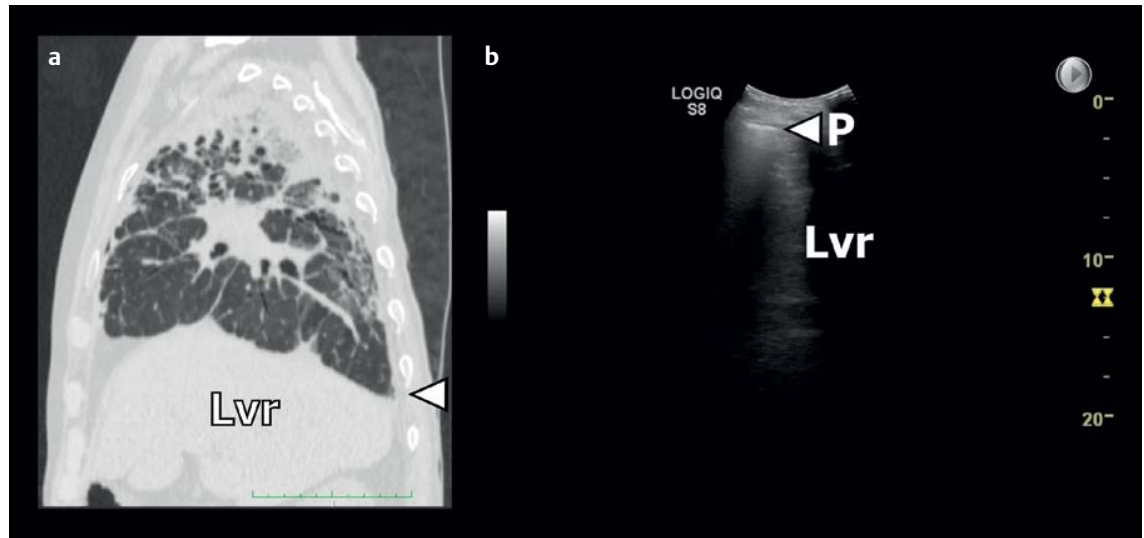

- Fig. 4 a: Sagittal high-resolution computed tomography (HRCT) image of the right lung and pleura showing pleural thickening and upper lobe fibrotic consolidation as part of pleuroparenchymal fibroelastosis (PPFE) related restrictive chronic lung allograft dysfunction ( $\mathrm{CCLAD}$ ). White arrow corresponds anatomically to LUS performed at the basal and posterior part of the right lower lobe, so-called zone R5, in b: LUS clip from the posterior and basal part of the right lower lobe showing no $B$-line artifacts (B) and a less thickened pleura without fragmentation (P). Lvr indicates the liver in both Figures $A$ and $B$.

2 Department of Respiratory Medicine and Allergology, Aarhus University Hospital, Aarhus, Denmark

3 Department of Cardiology, Rigshospitalet, Copenhagen University Hospital, Unit of lung transplantation, Copenhagen, Denmark

Correspondence

Dr. Jesper Rømhild Rømhild Davidsen Odense University Hospital Department of Respiratory Medicine Sdr. Boulevard 29

5000 , Odense $C$

Denmark

Tel.: +45/215/71 292, Fax: +45/661/10 127

jesper.roemhild.davidsen@rsyd.dk

Bibliography

ports the use of LUS as a potential monitoring tool for rCLAD (i. e., PPFE) progression.

In this case the supplemental LUS findings did not influence the clinical management but did provide the clinician with more specific knowledge regarding a worse prognosis than BOS. Such knowledge may ultimately provide better timing of overall management including long-term oxygen therapy, experimental treatments, re-transplantation, and palliation.

As there is no evidence on this topic, prospective studies validating LUS with HRCT findings as the gold standard in a series of cases with CLAD are warranted to yield more information about the role of LUS in a clinical context.

\section{Conflict of Interest}

The authors declare that they have no conflict of interest.

\section{Authors}

Jesper Rømhild Davidsen ${ }^{1}$, Christian Borbjerg Laursen ${ }^{1}$, Elisabeth Bendstrup², Hans Henrik Lawaetz Schultz ${ }^{3}$

\section{Affiliations}

1 Department of Respiratory Medicine, Odense University Hospital, Odense C, Denmark
DOI https://doi.org/10.1055/s-0043-116489 Ultrasound Int Open 2017; 3: E117-E119 (c) Georg Thieme Verlag KG Stuttgart . New York

ISSN 2199-7152

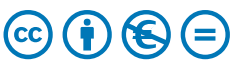

